\title{
Review of: "Supplementing cultured human myotubes with hibernating bear serum results in increased protein content by modulating Akt/FOXO3a signaling"
}

\author{
Hannah Hapner Hogan
}

Potential competing interests: The author(s) declared that no potential competing interests exist.

Miyazaki et al. explore the humoral effects of hibernating bear serum (HBS) on human skeletal muscle cells to investigate the anti-atrophy effects seen in hibernating Japanese black bears (Ursus thibetanus japonicus). Human myocytes were treated with HBS for 12 to 24 hours and protein content, quantified via puromycin incorporation, was measured. Notably, expression of specific protein degradation related proteins (MuRF-1) was downregulated post-treatment with HBS, indicating a potential regulation of the AKT/FOXO3a signaling pathway to prevent protein degradation and muscle atrophy.

This article further explores the unknowns of the effects of bear serum and the impacts it may have on cellular metabolism. I have a couple of comments. Firstly, in reference to the slightly (but recognized by the author) unsupported claim that FOXO3a sequestration to the cytosol, but not a decrease in the nuclear concentration, may lead to decreased transcriptional activity of FOXO3a. These results are very interesting, but I agree it may not be enough evidence to make any definitive statements. Exploration into the specific effects of increased overall FOXO3a phosphorylation and cytosolic sequestration and how it relates to nuclear activity seems a likely study to follow.

Additionally, it is important to keep in mind that the cells used here are human myocytes, not bear myocytes. As found previously in studies using bear adipocytes ([1][2][3]), bear cells are vastly different than human cells and respond to stimulus and treatments as such, indicating a humoral and intrinsic quality to the effects seen in vivo and in vitro. It may be more important to study both bear cells and serum together to elucidate what is driving these amazing adaptations rather than studying the effects bear serum has on human cells (at least for now).

\section{References}

1. Hannah R. Hapner Hogan, Brandon D. E. Hutzenbiler, Charles T. Robbins, Heiko T. Jansen. (2022). Changing lanes: seasonal differences in cellular metabolism of adipocytes in grizzly bears (Ursus arctos horribilis). J Comp Physiol B. doi:10.1007/s00360-021-01428-z. 
2. ' K. S. Rigano, J. L. Gehring, B. D. Evans Hutzenbiler, A. V. Chen, et al. (2016). Life in the fat lane: seasonal regulation of insulin sensitivity, food intake, and adipose biology in brown bears.J Comp Physiol B, vol. 187 (4), 649-676. doi:10.1007/s00360-016-1050-9.

3. `J. L. Gehring, K. S. Rigano, B. D. Evans Hutzenbiler, O. L. Nelson, et al. (2016). A protocol for the isolation and cultivation of brown bear (Ursus arctos) adipocytes. Cytotechnology, vol. 68 (5), 2177 2191. doi:10.1007/s10616-015-9937-y. 